\title{
Prevalence and Predictors of Gallbladder Stasis among Patients with Cholelithiasis
}

\author{
M Ravikiran ${ }^{1}$, Usha Dutta ${ }^{1 *}$, Anish Bhattacharya ${ }^{2}$, Kuldeep Solanki ${ }^{1}$, \\ GR Verma ${ }^{3}$, Sanjay Chaudhuri ${ }^{1}$, Rakesh Kochhar ${ }^{1}$, B Nagi ${ }^{1}$ and Kartar \\ Singh ${ }^{1}$ \\ ${ }^{1}$ Departments of Gastroenterology, Postgraduate Institute of Medical Education \\ and Research, Chandigarh, India \\ ${ }^{2}$ Departments of Nuclear Medicine, Postgraduate Institute of Medical Education \\ and Research, Chandigarh, India \\ ${ }^{3}$ Departments of Surgery, Postgraduate Institute of Medical Education and \\ Research, Chandigarh, India \\ *Corresponding Author: Usha Dutta, Professor, Departments of Gastroenterology, \\ Postgraduate Institute of Medical Education and Research, Chandigarh, India.
}

Received: May 25, 2021

Published: June 15, 2021

(C) All rights are reserved by Usha Dutta., et al.

\footnotetext{
Abstract

Background: GB stasis is an important cofactor in the pathogenesis of gallstones and gallbladder cancer. The prevalence and predictors of GB stasis among patients with GS in a high incidence area for GBC like North India is not known.

Aims: To determine the prevalence of gallbladder stasis among patients with GS using cholescintigraphy and to identify clinical, demographic and radiological predictors of GB stasis.

Methods: Patients with GS were prospectively evaluated for demographic, anthropometric, dietary and clinical parameters. Ultrasonography assessment for number and size of stones and to exclude associated malignancy/polyp was done. Gallbladder Ejection Fraction (GBEF) was assessed using HIDA scan after ingestion of fatty meal. GBEF of $\leq 35 \%$ was considered as evidence for GB stasis. Predictors of GB stasis were identified by univariate followed by multivariate analysis.

Results: 120 patients with GS were studied (mean Age $46 \pm 13$ years; 80\% F). Majority (92\%) were symptomatic, dyspepsia (78\%), biliary colic (70\%), postprandial discomfort (70\%), fat intolerance (31\%) or jaundice (5\%). Ultrasound showed multiple GS in 73\%. The median EF was 40\% (range 0 - 95\%). 47\% (56/120) had GB stasis \{non-visualized GB (39 cases) or low EF (17 cases)\}.

The median EF in the multiple GS group was lower than in single GS group [35\% vs 52\%; ( $p=0.021)$ ]. Patients with single GS were more likely to have good EF (> 75\%) than multiple GS group [12/33 (36\%) vs 10/87 (12\%); $p=0.002$ ]. GB stasis was found more often in the multiple GS group than single GS [45/87 (52\%) vs 11/33 (33\%) (p=0.07)]; among poor SES than upper SES group [44/84 (52\%) Vs 12/36 (33\%), ( $\mathrm{p}=0.05)]$; in those with postprandial discomfort than those without [44/84 (52.4\%) Vs 12/36 (33\%), ( $p=0.05)]$ and in those of sub-Himalayan belt than those from Punjab and Haryana [18/28 (64\%) vs 38/92 (41\%); $p=0.03]$. Independent risk factors for stasis were postprandial discomfort $(\mathrm{OR}=2.7 ; \mathrm{p}=0.03)$, belonging to sub-Himalayan belt $(\mathrm{OR}=4 ; \mathrm{p}=$ 0.007), poor SES (OR = 3.2; $\mathrm{p}=0.014$ ) and multiple GS (OR $=2.4 ; \mathrm{p}=0.05)$.

Conclusion: Gallbladder stasis is present in $47 \%$ of patients with GS. Independent predictors of GB stasis are presence of multiple GS, poor SES and post-prandial discomfort and in those belonging to states of sub-Himalayan belt.
}

Keywords: Gallstones; Gallbladder Stasis; Multiple Gallstones

Citation: Usha Dutta., et al. "Prevalence and Predictors of Gallbladder Stasis among Patients with Cholelithiasis". Acta Scientific Gastrointestinal Disorders 4.7 (2021): 40-46. 


\section{Introduction}

Cholelithiasis is a common clinical problem in most parts of the world accounting for significant morbidity and mortality. The prevalence of gallstones (GS) in the west is high (10\%) but the incidence of GBC is low $\left(0.1 / 10^{5}\right.$ population in USA). However, in India the prevalence of GS is only 3 - $5 \%$ but the incidence of GBC is high (13/10 $0^{5}$ population) [1-3]. Thus, there are possibly other cofactors operating along with GS to increase the risk for GBC. GB stasis is an important risk factor for the pathogenesis of GS as well as its symptomatology [4-6]. Patients with GS who have GB stasis are more likely to have recurrent biliary colic and less likely to respond to medical dissolution therapy and benefit from cholecystectomy [7-9]. In a recent study, we also found that GB stasis was also associated with the presence of incomplete intestinal metaplasia, a pre-malignant lesion of GB mucosa $[10,11]$. Patients with GS are at an increased risk for GBC and $60-90 \%$ of patients with GBC in India have associated GS $[12,13]$. Thus, gallbladder stasis may be an important cofactor in the pathogenesis of both GS and GB cancer in India. However, the prevalence of GB stasis in Indian patients with GS is not known.

North and North-eastern Indian states are high incidence areas for GBC compared to Southern India [14]. In North India, the sub-Himalayan belt comprising of states of Himachal Pradesh, Uttar Pradesh, Uttaranchal, West Bengal and Bihar comprise a high risk belt for GBC compared to the states of Punjab and Haryana. There are genetic and dietary differences between these populations. The prevalence of GB stasis, an important cofactor in the pathogenesis of GS and GBC, is not known. Also, expertise and facilities to estimate GB ejection fraction is not available in most hospitals of this region. Thus, determining simple predictors for GB stasis would be useful to identify the subgroup with GB stasis. GBC carries a dismal prognosis as the disease is far too advanced at presentation [15]. A cost effective strategy to prevent GBC may be to also perform early/ prophylactic cholecystectomy in patients with GS who are at high risk for GBC [12]. One of the high risk factors is GB stasis. Hence we planned a study to identify the prevalence and simple predictors of GB stasis among patients with GS.

\section{Methods}

A prospective study was conducted over a period of 3 years (2003 - 2006) in a tertiary care referral center in North India. Patients with symptomatic gallstone disease aged between 20 - 75 years were included. Patients with porcelain gall bladder, radiological evidence of GB malignancy, GB polyp, biliary obstruction, cystic duct obstruction, severely ill patients, patients on parenteral nutrition, pregnancy, history of rapid weight loss and those on opioids, calcium channel blockers or nitrates, estrogens/oral contraceptives, octreotide, known case of ileal disease/resection, cystic fibrosis, cirrhosis, hemolysis were excluded, as these are known to affect GB function. All the patients were assessed for age, gender, marital status, socioeconomic status, reproductive history, family history, dietary history and detailed symptom profile. The socio-economic class was classified according to the modified Kuppuswamy scale [16]. The patients belonging to the lower, upper lower and the lower middle class were categorized as poor SES and the upper class and upper middle class as higher SES. Anthropometric parameters including height, weight, body mass index, mid arm circumference and waist-hip ratio were also obtained in 74 patients. All were evaluated with a transabdominal ultrasonography for presence and number of GS, size of largest stone, presence of fatty liver, GB wall thickness and associated pathology.

Patients underwent hepatobiliary scintigraphy with ${ }^{99 \mathrm{M}} \mathrm{Techne-}$ tium labeled mebrofenin (BRIDA, bromo-2,4,6- trimethylacetanildo iminodiacetic acid) administered intravenously at a dose of $3 \mathrm{mCi}$ following standard guidelines $[17,18]$. Serial static images were acquired under a gamma camera (GE, USA) until activity was seen in GB. Patients were then given a standard fatty meal and repeated static images were obtained at specified intervals over 4 hours, beginning 45 min after the fatty meal [19]. The EF was calculated using a software after defining a region of interest around the GB in the pre-meal and post-meal images. GBEF values of less than $35 \%$ was taken as suggestive of stasis $[19,20]$. Absence of visualization of GB even after 4 hours of scan was classified as non-visualized GB and their effective $\mathrm{EF}$ was taken as $0 \%$.

Statistical analysis was done using Statistical Package for Social Sciences (SPSS) version 12. Descriptive data was expressed as mean \pm SD or as median, range as was appropriate. Univariate analysis was done to identify factors associated with GB stasis. Chi-square test, Student ' $t$ ' test, Kruskall-wallis test and Mann-Whitney $\mathrm{U}$ test were applied as was appropriate for the data under study and $\mathrm{p}$ value of $<0.05$ was considered as significant. Multivariate analysis was done to identify independent risk factors for gallbladder stasis in which we included those factors which were significant on univariate analysis with a $p$ value of $<0.1$.

\section{Results}

A total of 120 patients with GS were studied. The mean age was $46 \pm 13$ years. Females constituted $80 \%$ of the patients. Of the 120 
patients, 110 patients were detected to have GS while being evaluated for their dyspeptic symptoms and in the remaining 10 patients it was an incidental detection. The common symptoms were dyspepsia (78\%), biliary colic (70\%), postprandial discomfort (70\%) and fat intolerance $(31 \%)$. Past history suggestive of transient jaundice in $5 \%(6 / 120)$ of the patients. The median duration of dyspepsia was 12 months (1 - 180 months). Dyspepsia was present for more than 6 months in 54/120 patients (45\%). Recurrent biliary colic was present in 50 patients (42\%). These patients underwent cholecystectomy at the hospital without any major complications. In all these patients there was no radiological or histological evidence of malignancy.

\section{Demographic parameters}

Majority of them were residing in urban areas (73\%) and belonged to the states of Punjab and Haryana (92/120). The remaining patients belonged predominantly to the sub-Himalayan belt, states of Himachal Pradesh (16), Uttar Pradesh/Uttaranchal (5), Bihar/Jharkhand (2), Jammu Kashmir (2), West Bengal (2), Tibet (1). Most of the patients belonged to poor SES $(84 ; 70 \%)$ the rest $30 \%$ (36 patients) belonged to the higher SES. First degree relatives of 21 patients had history of GS. One patient had family history of gallbladder cancer. A history suggestive of typhoid fever in the past was obtained in 13 patients. History of smoking was present in 7 cases. There was history of alcohol abuse in non-cirrhogenic doses in 7 cases.

\section{Anthropometric parameters}

The mean height of all the patients with GS was $158 \pm 7 \mathrm{~cm}$ and the mean weight was $64 \pm 11 \mathrm{Kg}$. The mean body mass index was $25.3 \pm 4$ and the midarm circumference was $28.6 \pm 2.2 \mathrm{~cm}$. In the 74 patients in whom anthropometry was assessed 35 (47\%) were non-obese (BMI $\leq 25)$, 32 (43\%) were overweight and only 7 (9\%) were obese.

\section{Ultrasound finding}

On ultrasonography 87 patients (73\%) had multiple GS and 33 (27\%) had single gallstone. The size of the largest stone ranged from 4-30 $\mathrm{mm}$. Associated fatty liver was found in 10 patients (12\%). None of the patients had evidence of cystic duct obstruction by stone/features of acute cholecystitis or features of malignancy/GB polyp.

\section{Cholescintigraphy}

The gallbladder was visualized at a median time of 20 minutes (range 10 - 100) after injection of the dye and the intestine was vi- sualized at a median time of 15 minutes (5 - 120 minutes). Gallbladder was visualized in 81 patients (67.5\%). In 39 patients gallbladder could not be visualized despite 4 hours of scanning. Patients with non-visualized gallbladder were considered to have nonfunctional GB due to chronic cholecystitis. Of the 81 cases in whom GB EF could be calculated, 17 (14.16\%) had $\mathrm{EF} \leq 35 \%$ and 64 (53.34\%) had EF > $35 \%$. The median EF for all the cases was $40 \%$ (range 0 - 95\%) and the mean was $38.8 \% \pm 33.5 \%$. The mean EF among those with visualized gallbladder was $57.5 \% \pm 23.9 \%$ and median $\mathrm{EF}$ was $59 \%$ (range 10 - 95). Overall, 56 of the 120 patients (47\%) had evidence of GB stasis in the form of either non-visualized gallbladder (39 cases) or low EF (17 cases).

\section{Predictors of poor gallbladder function}

The mean age of patients with gallbladder stasis was similar to that of those with no gallbladder stasis $\{45.64 \pm 13$ vs. $45.97 \pm$ $12.7 ; \mathrm{p}=0.89\}$. The proportion of females in both the groups was also similar (79\% vs. $81 \%$; $\mathrm{p}=0.71$ ). Patients with multiple gallstones had a significantly lower median EF than those with single GS $[35 \%$ vs $52 \%$, $(p=0.021)]$. Patients with multiple GS, more often had GB stasis than those with single stone $[45 / 87$ (52\%) vs $11 / 33(33 \%)(p=0.07)]$. Patients with multiple GS were less likely to have good EF ( $>75 \%)$ than those with single GS [10/87 (12\%) vs 12/33 (36\%); $\mathrm{p}=0.002$ ].

Patients belonging to sub-Himalayan belt more often had GB stasis than those who belonged to Punjab and Haryana [18/28 (64\%) vs 38/92 (41\%); $p=0.033$ ]. Patients with GS from the sub-Himalayan states more often had non-visualized GB on cholescintigraphy as compared to those from $\mathrm{PH}[14 / 29$ (48.3\%) Vs $25 / 91(27.5 \%)(p=0.04)]$. Patients belonging to poor SES more often had GB stasis than those belonging to the upper SES [44/84 (52\%) Vs 12/36 (33\%), ( $p=0.05)]$.

Patients with postprandial discomfort more often had GB stasis than those without the symptom [44/84 (52\%) Vs 12/36 (33\%), $(\mathrm{p}=0.07)]$. Patients with recurrent biliary colic more often had stasis than those with one / no colic episode, however, the difference was not statistically significant (27/50 (54\%) vs $29 / 70$ (41\%); p $=0.174)$. The mean duration of gallstone disease in the GB stasis group was $20.49 \pm 31.5$ months in contrast to $15.8 \pm 17.4$ months in the normal EF group ( $p=n s$ ). There was no difference in the rural/ urban status of the patients' vis-à-vis the EF. None of the reproductive parameters, dietary, family history, anthropometric parameters studied was significantly different between the two groups (Table 1). 


\begin{tabular}{|l|c|c|c|}
\hline \multicolumn{1}{|c|}{ Parameters } & $\begin{array}{c}\text { GB stasis } \\
\text { (n = 56) }\end{array}$ & $\begin{array}{c}\text { No stasis } \\
\text { (n = 64) }\end{array}$ & p-value \\
\hline Age & $45.64 \pm 13$ & $45.97 \pm 12.7$ & 0.89 \\
\hline Female & $44(78.6 \%)$ & $52(81.3 \%)$ & 0.71 \\
\hline $\begin{array}{l}\text { Sub-Himalayan } \\
\text { belt }\end{array}$ & $18(32 \%)$ & $10(16 \%)$ & 0.03 \\
\hline $\begin{array}{l}\text { Poor socioeco- } \\
\text { nomic status }\end{array}$ & $44(78.6 \%)$ & $40(62.5 \%)$ & 0.05 \\
\hline $\begin{array}{l}\text { Postprandial } \\
\text { discomfort }\end{array}$ & $44(78.6 \%)$ & $40(63.5 \%)$ & 0.07 \\
\hline $\begin{array}{l}\text { Recurrent } \\
\text { biliary colic }\end{array}$ & $27(48 \%)$ & $23(36 \%)$ & 0.17 \\
\hline Fat intolerance & $21(37.5 \%)$ & $16(25.4 \%)$ & 0.15 \\
\hline Multiple stones & $45(80.4 \%)$ & $42(65.6 \%)$ & 0.07 \\
\hline
\end{tabular}

Table 1: Predictors of gallbladder stasis.

\section{Independent predictors of gallbladder stasis}

The factors significantly associated with GB stasis on univariate analysis were poor SES, post-prandial discomfort, state of origin and multiple GS. These four factors along with age and gender were studied using logistic regression analysis to identify independent predictors for GB stasis. The independent risk factors for GB stasis were postprandial discomfort $(\mathrm{OR}=2.7 ; \mathrm{p}=0.03)$, state of sub-Himalayan belt ( $O R=4 ; \mathrm{p}=0.007)$, poor SES $(\mathrm{OR}=3.2 ; \mathrm{p}=0.014)$ and multiple GS (OR $=2.4 ; \mathrm{p}=0.05)$. Gender and age were not found to be significant (Table 2).

\begin{tabular}{|l|c|c|c|c|}
\hline \multicolumn{1}{|c|}{ Parameters } & Odds ratio & $\begin{array}{c}\text { 95\% C.I. } \\
\text { lower limit }\end{array}$ & $\begin{array}{c}\text { 95\% C.I. } \\
\text { upper } \\
\text { limit }\end{array}$ & p value \\
\hline $\begin{array}{l}\text { Sub- Himalayan } \\
\text { belt }\end{array}$ & 3.96 & 1.47 & 10.7 & 0.007 \\
\hline Poor SES & 3.22 & 1.268 & 8.188 & 0.01 \\
\hline $\begin{array}{l}\text { Multiple } \\
\text { gallstones }\end{array}$ & 2.43 & 0.986 & 5.983 & 0.05 \\
\hline $\begin{array}{l}\text { Postprandial } \\
\text { discomfort }\end{array}$ & 2.71 & 1.096 & 6.74 & 0.03 \\
\hline Age & 1.002 & 0.971 & 1.033 & 0.91 \\
\hline Male & 1.31 & 0.487 & 3.54 & 0.59 \\
\hline
\end{tabular}

Table 2: Independent predictors of gallbladder stasis.

\section{Discussion}

In our study conducted on 120 patients with GS, we found that nearly half (47\%) of patients had evidence of gallbladder stasis and

\section{PREDICTORS FOR GB STASIS}

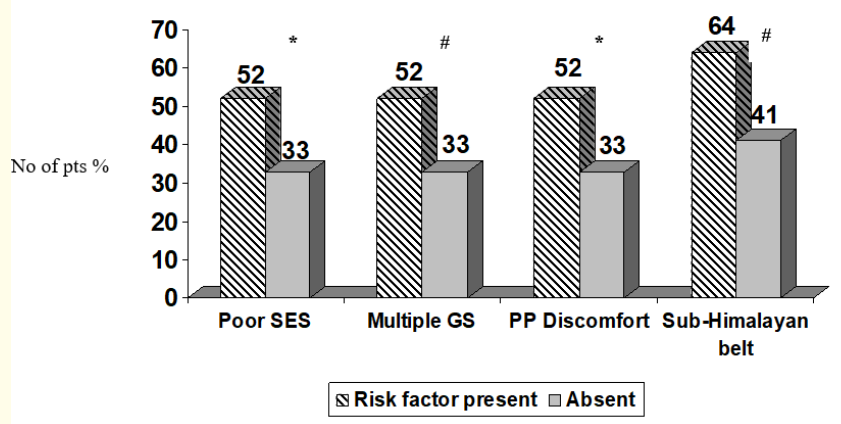

Figure 1: Risk factors for gallbladder stasis: This bar diagram shows that GB stasis was more often in patients with multiple GS, patients of poor SES, in those with symptoms of postprandial discomfort or among those belonging to the sub-Himalayan belt. The hatched bar indicates presence of risk factors and the grey plain bar indicates absence of risk factors. The $p$ values are indicated above the graphs ("*” indicates $\mathrm{p}=0.05$ and “\#” indicates $\mathrm{p}=0.07$ ).

one third (33\%) had non-visualized gallbladder on cholescintigraphy. The independent predictors of GB stasis were presence of multiple GS, history of postprandial discomfort, poor SES and ethnic origin from North Indian states of sub-Himalayan belt.

This is the first large study to evaluate the prevalence and predictors of GB stasis among patients with GS. There is no study from India on this issue, where GS and GBC are fairly prevalent. There is only one study in literature on 26 patients by Pomeranz and Shaffer, which attempted to identify factors associated with GB stasis [21]. However, the authors could not draw any significant conclusions due to very small sample size.

There was no effect of age on GBEF. The mean age of patients with GB stasis was similar to those with normal EF. Age and EF showed no correlation ( $r h o=-.007, p=0.943$ ). A study on gallbladder motility in young vs elderly had shown that there was no difference in EF between the two groups [22]. Our study population had predominantly $(80 \%)$ females. This is similar to the pattern observed elsewhere as GSD is primarily a disease of women [4]. Women have 2.7 times higher age adjusted odds for GS compared to men due to the hormonal influences on the GB [4]. 
The symptom profile in our study group was similar to other study groups [23]. Biliary colic, postprandial discomfort and dyspepsia are the commonest symptoms in also other series [4]. Nonvisualization of the GB on hepatobiliary scintigraphy in our patients was due to chronic cholecystitis resulting in poor GB function [24]. Other recognized causes of non-visualized GB like acute cholecystitis, cystic duct obstruction, prolonged fasting (> 24 hours), severely ill patients, patients receiving parenteral nutrition, presence of severe hepatobiliary disease, insufficient fasting ( $<4$ hours), pancreatitis, previous cholecystectomy were absent in our patients [24].

Nearly half of the patients with GS had evidence of GB stasis and one-third had a non-functioning GB. GB stasis has been found to be associated with a greater risk for pre-malignant lesions in the GB in a recent study from our group [10]. Accumulation of toxins and metabolites in the bile may probably result in chronic mucosal injury resulting in mutagenesis, metaplasia and dysplasia. Aggrawal., et al. showed that the biliary malonaldehyde (a toxin) levels was higher in those with GB stasis [25]. Thus, GB stasis may be a hitherto unidentified and yet fairly prevalent cofactor in the pathogenesis of GBC in India. The other identified cofactors for GBC in India are older age, chronic Salmonella typhi carrier state, smoking, poor SES and high parity [13].

Interestingly, since GB stasis is prevalent in nearly half of the GS population. Thus, the attributable risk of this factor may be higher than the other cofactors for GBC. The incidence of GBC in India is highest in the sub-Himalayan belt, which runs from north to northeastern India which comprises the states of Uttar Pradesh, Uttaranchal, Himachal Pradesh, West Bengal, Bihar and Jharkhand $[14,26,27]$. In contrast, the Punjab and Haryana states have a lower incidence of GBC, despite a high prevalence for gallstones. GB stasis was found in our study to be more prevalent in the sub-Himalayan belt than the Punjab/Haryana states suggesting that it may be a cofactor in the pathogenesis of GBC in this region. Those from sub-Himalayan high risk belt had significantly more GB stasis and more often had non-visualized GB [14/29 (48.3\%) Vs 25/91(27.5\%) (p $=0.037)]$. We found that people living in Punjab and Haryana more often were non-vegetarians [61/92 (63\%) vs 11/28(39\%); $\mathrm{p}=$ 0.01 ] which may indicate better and higher quality protein intake. In a recent study, we found that poorer ejection fraction was found in patients consuming diets rich in fats but poor in proteins [29]. Combination of high fat and low protein intake in sub-Himalayan belt may account for their poorer GB function. The ethnic origin of people of Punjab/Haryana is different and hence they are ge- netically different than those of the sub-Himalayan states. There were no significant differences in the anthropometry, age, gender and other demographic and clinical parameters between these two groups. Thus, ethnic origin and differences in diet possibly account for differences in risk for stasis and GBC.

Postprandial discomfort was found to be an independent predictor of GB stasis. Inadequate and mistimed bile delivery may result in maldigestion of food with resultant postprandial discomfort. Recurrent biliary colics ( $>1$ episodes) was associated with non-visualized GB and GB stasis. Biliary colics may result in inflammation induced destruction of the GB neuro-muscular apparatus, which results in loss of GB function over a period of time. Hong SN., et al. on 31 patients with GS showed that abnormal GBEF was associated with recurrence of pain [8]. However, in the study by Pomeranz and Shaffer, no relationship was found between biliary colic and GB function [21].

Presence of multiple GS was another independent predictor of GB stasis. Multiple stones are more likely in those with stasis as stasis is a risk factor for stone disease. Also, multiple stones are more likely to be associated with more GB inflammation, persistent/recurrent infection which in turn may affect GB function [11]. Stasis itself also may perpetuate growth in size and number of stones. Patients with single stones more often had normal GB function. Single gallstone may represents an early stage in the evolution of gallstone disease when GB is still contracting well and pushing out the sludge and small stones which are getting formed. Moreover, single stones may also be pure cholesterol stones where the main pathogenetic pathway is supersaturated bile rather than gallbladder hypomotility [28].

Poor socio-economic status was found to be an independent predictor of GB stasis as well as non-visualized gallbladder. This may be related to malnutrition, inadequate dietary protein intake, intake of protein of poor biological value and deficiency of micronutrients and antioxidants in the diet [30,31]. Also, people belonging to poor SES tend to ignore colic episodes because of cost considerations or higher pain threshold and resort to household remedies. Because of associated factors like poor access to education and modern healthcare facilities, they tend to present late to modern health care system. Patients belonging to poor SES more often had non-visualized GB at the time of their presentation than those belonging to the upper SES (31/84 (37\%) vs 8/53 (15\%); $\gamma=$ 0.09 ) indicating a more advanced disease. 
Obesity is a risk factor for gall stone formation in the west and is usually associated with supersaturation of the bile with cholesterol [28]. However, in our study, most of the patients were non-obese. However, GB hypomotility was very common. Thus, hypomotility rather than cholesterol supersaturation may be the dominant factor in the pathogenesis of GS in India. We found that the mean protein intake in our study patients was $45 \pm 10$ gms/day and most of the patients were consuming lower than the recommended dietary allowance (60 gm/day). As Indians consume inadequate amount of protein in their diet and the sources are of poor biological value compared to the west, this may reflect in poor GBEF in Indians and thus placing them at a higher risk for GBC and GS despite absence of obvious obesity.

This is the first study, which has addressed the issue of prevalence of gallbladder stasis in Indian patients with GS and its predictors. Our study has shown that GB stasis is prevalent in nearly half of the patients with GS in India. The predictors for GB stasis are presence of multiple GS, poor SES, ethnic origin from sub-Himalayan belt and presence of postprandial discomfort. Identification of this high-risk group for GB stasis will help us in the understanding the pathogenesis of GBC and also allow us to target interventions for preventing gallbladder cancer. Patients with GB stasis are more likely to have pre-malignant lesions in the GB, hence early cholecystectomy and detailed examination of the GB specimen at histology may help in reducing the incidence, morbidity and mortality of gallbladder cancer in India.

\section{Conclusion}

Gallbladder stasis is present in $47 \%$ of patients with GS. Independent predictors of GB stasis are presence of multiple GS, poor SES and post-prandial discomfort and in those belonging to states of sub-Himalayan belt.

\section{Bibliography}

1. Kratzer W., et al. "Prevalence of gallstones in sonographic surveys worldwide". Journal of Clinical Ultrasound 27 (1999): 1-4.

2. Tandon RK. "Studies in pathogenesis of gallstones in India". Annals of the National Academy of Medical Sciences 25 (1989): 213-222.

3. Singh V., et al. "Epidemiology of gallstone disease in Chandigarh: a community-based study". Journal of Gastroenterology and Hepatology 16 (2001): 560-563.
4. The epidemiology of gallstone disease in Rome, Italy. Part II: Factors associated with the disease. The Rome Group for Epidemiology and Prevention of Cholelithiasis (GREPCO)". Hepatology 8 (1988): 907-913.

5. Tandon RK., et al. "Increased incidence of biliary sludge and poor gallbladder contractility in patients with high spinal cord injury". Gut 41.5 (1997): 682-687.

6. Quigley EM., et al. "Hepatobiliary complications of total parenteral nutrition". Gastroenterology 104.1 (1993): 286-301.

7. Chan DC., et al. "Gallbladder contractility and volume characteristics in gallstone dyspepsia". World Journal of Gastroenterology 10 (2004): 721-724.

8. Hong SN., et al. "Usefulness of gallbladder ejection fraction estimation to predict the recurrence of biliary pain in patients with symptomatic gallstones who did not undergo cholecystectomy". Digestive Diseases and Sciences 49 (2004): 820-827.

9. Spengler U., et al. "Gallbladder motility before and after extracorporeal shock-wave lithotripsy". Gastroenterology 96 (1989): 860-863.

10. Choudhury S. "To study the effect of GB stasis on GB histology among patients with symptomatic gallstones undergoing cholecystectomy". (MD thesis) submitted to PGIMER, Chandigarh, India (2004).

11. Latio M. "Intestinal, gastric body and antral type mucosal metaplasia in the gallbladder". Beitr Pathol Bd 159 (1976): 271-279.

12. Mohandas KM and Patil PS. "Cholecystectomy for asymptomatic gallstones can reduce gall bladder cancer mortality in northern Indian Women". Indian Journal of Gastroenterology 25 (2006): 147-151.

13. Dutta U., et al. "Typhoid carriers among patients with gallstones are at an increased risk for carcinoma gallbladder". The American Journal of Gastroenterology 95 (2000): 784-787.

14. Nandakumar A., et al. "Geographic pathology revisted: Development of an atlas of cancer in India". International Journal of Cancer 116 (2005): 740-754.

15. Batra Y., et al. "Gallbladder Cancer in India: a dismal picture". Journal of Gastroenterology and Hepatology 20 (2005): 309314. 
16. Park JE and Park K. "Text book of Preventive and Social medicine". Banarasidas Bhanot, Jabalpur, $13^{\text {th }}$ edition (1991): 160162.

17. Vyas PK., et al. "Estimation of gallbladder ejection fraction utilizing cholecystokinin - stimulated magnetic resonance cholangiography and comparisons with hepatobiliary scintigraphy". Journal of Magnetic Resonance Imaging 15 (2002): 78-81.

18. Siegal A., et al. "Gallbladder ejection fraction: Correlation of scintigraphic and ultrasonographic techniques". Clinical $\mathrm{Nu}$ clear Medicine 25 (2000): 1-6.

19. Krishnamurthy GT and Brown PH. "Comparison of fatty meal and intravenous cholecystokinin infusion for gallbladder ejection fraction". The Journal of Nuclear Medicine 43 (2002): 1603-1610.

20. Fink-Bennett D., et al. "Cholecystokinin cholescintigraphy: detection of abnormal gallbladder motor function in patients with chronic acalculous gallbladder disease". The Journal of Nuclear Medicine 32 (1991): 1695-1699.

21. Pomeranz IS and Shaffer EA. "Abnormal gallbladder emptying in a subgroup of patients with gallstones". Gastroenterology 88 (1985): 787-791.

22. Rajan M., et al. "Ultrasonographic assessment of gall bladder kinetics in the elderly". Indian Journal of Gastroenterology 19 (2000): 158-160.

23. Berhane T., et al. "Pain attacks in non-complicated and complicated gallstone disease have a characteristic pattern and are accompanied by dyspepsia in most patients: the results of a prospective study". Scandinavian Journal of Gastroenterology 41 (2006): 93-101.

24. Balon HR., et al. "Procedure guideline for hepatobiliary scintigraphy". The Journal of Nuclear Medicine 38 (1997): 1654-1657.

25. Aggrawal M., et al. "An USG evaluation of gallbladder emptying in patients with cholelithiasis". Journal of Clinical Gastroenterology 31 (2000): 309-313.

26. Dhir V and Mohandas KM. "Epidemiology of digestive tract cancers in India IV. Gall bladder and pancreas". Indian Journal of Gastroenterology 18 (1999): 24-28.
27. National Cancer Registry Programme. "Consolidated report of the population based cancer registries 1990-1996". Incidence and distribution of cancer. New Delhi: Indian Council of Medical Research (2001): 52-53.

28. Ho KJ., et al. "Bile acid pool size in relation to functional status of gallbladder and biliary lipid composition in Chinese". The American Journal of Clinical Nutrition 33 (1980): 1026-1032.

29. Solanki K. "Effect of Iron deficiency on gallbladder motility: a cross-sectional study in postpartum patients". DM dissertation to PGIMER, Chandigarh (2005).

30. Chandra RK. "Nutrition and immunoregulation. Significance for host resistance to tumours and infectious diseases in humans and rodents". Journal of Nutrition 122.3 (1992): 745757.

31. Dutta U., et al. "Patients with gallstones develop gallbladder cancer at an earlier age". European Journal of Cancer Prevention 14 (2005): 381-385.

Volume 4 Issue 7 July 2021

(C) All rights are reserved by Usha Dutta., et al. 